PROCEEDINGS OF THE

AMERICAN MATHEMATICAL SOCIETY

Volume 130, Number 3, Pages 723-726

S 0002-9939(01)06077-4

Article electronically published on July 25, 2001

\title{
SURVIVAL OF THE WEAK IN HYPERBOLIC SPACES, A REMARK ON COMPETITION AND GEOMETRY
}

\author{
ITAI BENJAMINI
}

(Communicated by Claudia M. Neuhauser)

\begin{abstract}
A simple competition model is presented. While in Euclidean spaces the weak will "die out", in the presence of hyperbolicity, coexistence take place.
\end{abstract}

\section{INTRODUCTION}

In this note we discuss a simple model of competition between two deterministically growing clusters, according to local rules. One cluster grows faster than the other. This model can be defined either discretely on a graph or in a continuous version on manifolds. For simplicity we will consider the discrete model. On Euclidean spaces or lattices, the faster cluster will eventually surround the slower cluster. We will see that this is not the case on Gromov hyperbolic spaces. The geometric properties of hyperbolic spaces are manifested in a very clear way in the behaviour of the model. At the end of the note we further remark about competition and geometry. We now describe the model.

Let $G=(V, E)$ be a graph, denote by $d$ the graph metric on $G$, and consider the following competition, between $X$ and $Y$, over the vertices of $G$.

Let $m$ be some positive integer, and let $x_{0}, y_{0}$ be two vertices in $V$. Set $X_{0}=$ $\left\{x_{0}\right\}, Y_{0}=\left\{y_{0}\right\}$. Inductively, let $X_{j+1}$ be the set of vertices $v$ that are not in $Y_{j}$ and satisfy $d\left(v, X_{j}\right) \leq m$ and let $Y_{j+1}$ be the set of vertices $v^{\prime}$ that are not in $X_{j+1}$ and satisfy $d\left(v^{\prime}, Y_{j}\right) \leq 1$. Finally, set $Y=\bigcup_{j=0}^{\infty} Y_{j}, X=\bigcup_{j=0}^{\infty} X_{j}$.

Must the set $Y$ be bounded? Must it be unbounded? Or does the result depend on the location of $x_{0}, y_{0}$ ?

The growth process was set so that the $X$ cluster grows $m$ times faster than the $Y$ cluster (and even win in a tie situation). It is easy to see that on $\mathbf{Z}^{d}, d>1$, assuming $m>1$, eventually the $X$ cluster will surround the $Y$ cluster. If $m=1$, both $X$ and $Y$ will have infinite volume, i.e. coexistence occurs. We will see below that for any $m$ on any Gromov hyperbolic graph which contains a bi-infinite geodesic, coexistence is guaranteed, once the initial position is chosen properly. We now define hyperbolic graphs. First, recall that a geodesic in a graph is a path for which the distance between any two vertices on the path is equal to the length of the path between these two vertices.

Received by the editors May 20, 2000 and, in revised form, August 25, 2000.

1991 Mathematics Subject Classification. Primary 30F45, 82C99.

(C)2001 American Mathematical Society 
Definition ( $\delta$-hyperbolic). Let $G=(V, E)$ be a graph. Given three vertices $u, v, w$ $\in V$, pick geodesics between any two to get a geodesic triangle. Denote the geodesics by $[u, v],[v, w],[w, u]$ and by $d$ the graph metric on $G$. Say the triangle is $\delta$-thin if for any $v^{\prime} \in[u, v]$

$$
\min \left(d\left(v^{\prime},[w, u]\right), d\left(v^{\prime},[v, w]\right)\right) \leq \delta,
$$

and the same for $v^{\prime} \in[w, u]$ or $[v, w]$.

$G$ is said to be $\delta$-hyperbolic if all geodesic triangles in $G$ are $\delta$-thin. Let

$$
\delta(G)=\inf \{\delta \mid G \text { is } \delta \text {-thin }\} .
$$

$G$ is Gromov hyperbolic if $\delta(G)<\infty$.

Trees are the most simple examples of Gromov hyperbolic graphs, but there are more interesting examples such as lattices in the real hyperbolic space $\mathbf{H}^{n}$. It is easy to see that Euclidean lattices are not hyperbolic. See Canon, Floyed, Kenyon and Parry (1997) (available on-line from MSRI) for an introduction to hyperbolic geometry and real hyperbolic spaces. There are many recent publications on hyperbolic metric spaces and graphs. The following graph provides a simple discrete approximation for the hyperbolic plane.

Example. Consider the graph obtained by adding to the binary tree edges connecting all vertices of the same level along a line. That is, represent the vertices of the binary tree by sequences of zeros and ones in the usual way. Add to the binary tree an edge between $v, w$ if $v$ and $w$ are at level $n$ and $|0 . v-0 . w|=1 / 2^{n}$, where $0 . v$ is the number in $[0,1]$ presented by the sequences corresponding to $v$.

The competition model above is a deterministic trivialization of a model for competing spatial growth, introduced by Häggström and Pemantle (1998), which is built on first passage percolation. In a sense their result can be viewed as showing coexistence in the deterministic model on a random perturbation of the Euclidean planar lattice, when $X$ and $Y$ grow with the same speed. Ancona (1988) studied the Martin boundary of Gromov hyperbolic graphs. Asymptotic properties of the Green function or other related random processes on hyperbolic graphs manifests nicely geometric properties of these graphs. The coexistence result has a similar flavor and the proof is based on a similar, yet much simpler, reasoning.

Theorem 1. Assume $G$ is a Gromov hyperbolic graph, which contains a bi-infinite geodesic $\gamma$. Then if $x_{0}$ and $y_{0}$ are both on $\gamma$, and $d\left(x_{0}, y_{0}\right)$ is sufficiently large, then half $\gamma$ is in $\bigcup_{k} X_{k}$ and half is in $\bigcup_{k} Y_{k}$.

\section{Proof AND FURTher REMARKS}

Proof of Theorem 1]. Pick a base point in $\gamma$, denoted $\gamma(0)$, and identify the vertices of $\gamma$ as $\gamma(n), n \in \mathbf{Z}$, in the obvious way. For $x$ real, let $\gamma(x)=\gamma(n(x))$, where $n(x)$ is the closest integer to $x$. Let $x_{0}=\gamma(0)$, and let $y_{0}=\gamma(n)$, for $n$ that will be determined later. Let $C(n)$ be the cone of all vertices $x$, for which any geodesic $\gamma^{\prime}$ from $\gamma(0)$ to $x$ satisfies $d\left(\gamma(n), \gamma^{\prime}\right)<2 \delta, \delta$ a fixed integer bigger than $\delta(G)$. By Ancona (1988) Theorem 6.9, for any geodesic $\gamma^{\prime \prime}$ from $y \in G-C(n-4 \delta)$ to $x \in C(n), d\left(\gamma(n), \gamma^{\prime \prime}\right)<2 \delta$. We need another standard fact (Gromov (1987) 7.1.A), namely that if $z$ is a vertex on a geodesic between $x$ and $y$, then the length of any path from $x$ to $y$, which is disjoint from a ball of radius $r$ centered at $z$, is larger than $c^{r}$ for $c$ depending only on $\delta$. 
By time $n$, which bounds from below the time taken for the $X$ cluster to reach $C(n)$, the $Y$ cluster reaches $\gamma(n+n / m)$, as well as a ball of radius larger than $r_{1}=n /(2 m)$ centered at $\gamma(n)$. The distance between any vertex in $G-C(n)$ to $C(n+n / m)$, outside that ball around $\gamma(n)$, is larger than $c^{r_{1}}$ for $c>1$, depending on $\delta$ only. So by the time the $X$ cluster will reach $C(n+n / m)$, if at all, the $Y$ will reach $\gamma\left(n+n / m+c^{r_{1}} / m\right)$, and will cover a ball of radius $c^{r_{1}} / 2 m$ around $\gamma(n+n /(2 m))$. To reach $C\left(n+n / m+c^{r_{1}} / m\right)$, outside the $Y$ ball, centered at $\gamma(n+n /(2 m))$, the $X$ cluster will need more than $c^{c^{r_{1}} / 2 m}$ steps. Thus, once $n$ is chosen so that $c^{r_{1}}=c^{n / 2 m}>n$, so $c^{c^{r_{1}} / 2 m}>c^{r_{1}}>n$, we can proceed as in the first stage, again and again, and the $X$ cluster will never reach $\bigcup_{k \geq n} \gamma(k)$.

Remarks. 1. It is clear from the robustness of the proof that a similar proof gives coexistence for the randomize version of Häggström and Pemantle (1998), for any two rates, on any Gromov hyperbolic graph.

2. Given $m$, the distance between the starting points, which is sufficient for coexistence, provides a measure of the hyperbolicity.

3. Note that Cayley graphs of finite extensions of free products (though not always hyperbolic) contains bi-infinite geodesics, which intersects infinitely many translations of a fixed finite cut set, hence coexistence holds for any $m$. Maybe one can identify the Cayley graphs, for which coexistence holds, for all $m$. For Cayley graphs with one end, maybe hyperbolicity is also necessary. Note that on any graph which is a product of two infinite graphs, coexistence does not hold. In particular on the product of a binary tree and a line, coexistence fails. This shows that exponential volume growth is not sufficient for coexistence.

4. Consider a variant in which the growth rate $m$ is changing in time. Then provided $m=m(n)$ grows slower than $a^{n}$, for some $a$ depending only on $\delta$, coexistence holds (for some starting vertices) by the same proof.

5. On Euclidean lattices, assuming $m>1$, the slower cluster is finite. It seems of interest to identify the shapes of these finite clusters, as in particular they might appear in real world phenomena.

6. Modern day competition (over the internet) should be modeled on more connected graphs, e.g. expanders, the hypercube or complete graphs, rather than on Euclidean or hyperbolic lattices.

Example. A continuous time symmetric competition. Start with one red vertex and one blue vertex on a graph with $n$ vertices. Each vertex is colored once and keep that color. Color a vertex red at exponential time with rate

\section{(number of red neighbouring vertices) $^{a}$}

for some power $a$, and the same for the blue. (The case $a=2$ can be interpreted as a sexual reproduction model.) On the complete graph, one can see that if $a>1$, then one color will take over all but a tight number of vertices. In fact, this becomes equivalent to some urn model problems; see Pemantle (1990). For $a=1$ on $\mathbf{Z}^{2}$, Häggström and Pemantle (1998) proved coexistence. It would be nice to verify coexistence for some $a>1$ on $\mathbf{Z}^{2}$, and to look at competitions on other graphs as well, for instance $G(n, p(n))$.

Exercise. Try to imagine evolution in hyperbolic space.... 


\section{ACKNOWLEDGMENTS}

Thanks to Olle Häggström and Oded Schramm.

\section{REFERENCES}

[1] A. Ancona, (1988), Positive harmonic functions and hyperbolicity, LNM 1344 Springer, 1-23. CMP 21:05

[2] J. Canon, W. Floyed, R. Kenyon, R. and W. Parry, (1997), Hyperbolic geometry, Math. Sci. Res. Inst. Publ. 31 59-115. MR 99c:57036

[3] M. Gromov, (1987), Hyperbolic groups, Math. Sci. Res. Inst. Publ. 8, Springer, 75-263. MR 89e:20070

[4] O. Häggström and R. Pemantle, (1998), First passage percolation and model for competing spatial growth, J. Appl. Prob. 35, 683-692. MR 2000f:60153

[5] R. Pemantle (1990), A time-dependent version of Polya's urn, J. Theoret. Prob. 3 627-637. MR 91i:60030

Department of Mathematics, The Weizmann Institute of Science, Rehovot, Israel 76100

E-mail address: itai@wisdom.weizmann.ac.il

$U R L:$ http://www.wisdom.weizmann.ac.il/〜itai/ 